\title{
The Motion of Kaluza-Klein Monopoles
}

\author{
P.J. Ruback \\ Department of Applied Mathematics and Theoretical Physics, Silver Street, \\ Cambridge CB3 9EW, United Kingdom
}

\begin{abstract}
A scheme is proposed and justified for examining the motion of the five dimensional Kaluza-Klein monopoles at low energy. The classical and quantum scattering is discussed and it is shown that for all separations and at small velocities the monopoles do not interact with one another.
\end{abstract}

\section{Introduction}

There has recently been progress in discovering the approximate motion of solitons arising in field theories, Yang-Mills-Higgs theory [1] and the $\mathbb{C} P^{1}$ model [2]. This is of interest for two reasons; first, if one adopts the view that solitons may correspond to physical particles, then one has information on their dynamics and their interactions. Secondly, in the light of the conjectured dualities between solitons as non-perturbative phenomena and perturbative quanta in field theories, such information would either corroborate or alternatively completely eliminate the possibility of such a duality. These recent results have been obtained by constructing approximate time independent solutions of the field equations, which are successions of exact static solutions, the quasistatic approximation. The motion is geodesic on the space of static $N$-soliton solutions equipped with a natural metric.

The aim of this article is to extend this scheme to the solitons of a gravity theory - five dimensional Kaluza-Klein theory [3] - and is organised as follows. In Sect. 2 the monopoles of Gross, Perry, and Sorkin [4] are described. In Sect. 3 the space of gravitational degrees of freedom is discussed and the moduli space of $\mathrm{N}$-monopole metrics is obtained. In Sect. 4 the approximation of geodesic motion on the moduli space is justified and in Sect. 5 the metric is constructed. Section 6 discusses the application of the metric to the problem of classical and quantum scattering and the accuracy of the scheme is considered. Section 7 concludes the article.

\section{Geometry of the Kaluza-Klein Monopoles}

Simple Kaluza-Klein theory is five dimensional General Relativity with the restriction that the fith dimension is given by a circle. That is, the coordinate $x^{5}$ is 
identified modulo $2 \pi R$, where $R$ is very small. We may express the five dimensional metric, $g_{A B}$ as a Fourier series in $x^{5}$;

$$
g_{A B}\left(x^{\alpha}, x^{5}\right)=\sum_{n=-\infty}^{n=\infty} g_{A B}^{n}\left(x^{\alpha}\right) \exp \left(\text { in } x^{5} / R\right) \quad \alpha=0,1,2,3 .
$$

All modes with $n \neq 0$ have energies greater than $h c / R$, and so the effective low energy theory is described solely by the $n=0$ mode. Thus we may neglect any $x^{5}$ dependence and therefore write the five dimensional line element as

$$
\begin{aligned}
d s^{2}= & -\exp (4 \kappa \sigma / \sqrt{3})\left(d x^{5}+2 \kappa A_{\alpha} d x^{\alpha}\right)^{2} \\
& +\exp (-2 \kappa \sigma / \sqrt{3})^{4} g_{\alpha \beta}\left(x^{\gamma}\right) d x^{\alpha} d x^{\beta}, \quad \alpha, \beta, \gamma=0,1,2,3 .
\end{aligned}
$$

The five dimensional Einstein equations for this ansatz can be obtained from a four dimensional effective action

$$
\int d^{4} x \sqrt{-{ }^{4} g}\left(-\frac{R}{4 \kappa^{2}}-\frac{1}{4} \exp (2 \sqrt{3} \kappa \sigma) F_{\alpha \beta} F^{\alpha \beta}+\frac{1}{2}^{4} g^{\alpha \beta} \partial_{\alpha} \sigma \partial_{\beta} \sigma\right),
$$

where $R$ is the scalar curvature of the metric ${ }^{4} g_{\alpha \beta}$. So we have Einstein Maxwell theory coupled to a scalar field $\sigma$ in a non-trivial manner, and we identify $\kappa^{2}=4 \pi G$, where $G$ is Newton's constant in four dimensions.

If we seek time independent solutions of the form

$$
d s^{2}=d t^{2}-{ }^{4} g_{\mu v} d x^{\mu} d x^{\nu} \quad \mu, v=1,2,3,5,
$$

then ${ }^{4} g_{\mu \nu}$ must satisfy the four dimensional Euclidean Einstein equations and possess a Killing vector $\frac{\partial}{\partial x^{5}}$. In addition, in order that the internal space with coordinate $x^{5}$ be given by a circle, the constant time hypersurfaces must have the topology of an $S^{1}$ bundle over some base space. If our solution is to tend asymptotically to the vacuum of Kaluza-Klein theory, that is $S^{1} \times$ Minkowski space, then the hypersurfaces must have the topology of an $S^{1}$ bundle over $S^{2} \times R$ at infinity. In other words ${ }^{4} g_{\mu \nu}$ describes an asymptotically locally flat (ALF) gravitational instanton. We can choose as our solutions the Multi Taub-NUT metrics [5]

$$
{ }^{4} g_{\mu v} d x^{\mu} d x^{v}=U \mathbf{d x} \cdot \mathbf{d x}+U^{-1}\left(d x^{5}+2 \kappa \mathbf{A} \cdot \mathbf{d x}\right)^{2},
$$

where

$$
U=1+4 G M \sum_{I=1}^{I=M} \frac{1}{\left|\mathbf{X}-\mathbf{X}^{I}\right|}=1+\sum_{I=1}^{I=N} U^{I}
$$

and

$$
\nabla U=2 \kappa \nabla \times \mathbf{A} .
$$

Clearly $\mathbf{A}$ is defined only up to $\mathbf{A} \rightarrow \mathbf{A}+\nabla \phi$, but by a coordinate transformation $x^{5}$ $\rightarrow x^{5}-2 \kappa \phi$, we obtain the same metric. Thus we are able to transform away the Dirac strings which arise, by covering the manifold with different patches in the northern and southern hemispheres of the points $\mathbf{X}^{I}$. These points themselves are non-singular if we identify $0 \leqq x^{5} \leqq 4 \kappa^{2} M$. 
Viewed from five dimensions these solutions are the monopoles of Gross, Perry and Sorkin which may be regarded as the solitons of the theory. The Maxwell field $A_{\alpha}$ is that of a collection of electrically neutral, magnetically charged static monopoles at positions $\mathbf{X}^{I}$ with moments $P=2 \kappa M$. In addition we can identify a scalar charge. If a scalar field $\phi$ behaves like

$$
\phi \approx \phi_{0}+\frac{\Sigma_{\phi}}{r} \text { as } r \rightarrow \infty
$$

then we call $\Sigma_{\phi}$ the scalar charge of the field, as on examining the expression for the energy of the superposition of two such static sources, we find that they attract each other with an inverse square law force proportional to the product of their charges. Here we see that each monopole has scalar charge $\Sigma=-\sqrt{3} G M$ and mass $M$, and we have the equality

$$
M^{2}+\frac{\Sigma^{2}}{G}-\frac{P^{2}+Q^{2}}{\kappa^{2}}=0,
$$

that is, the gravitational and scalar attraction is equal and opposite to the magnetic repulsion. This is Scherk's "antigravity" condition [6] and explains why we can place the monopoles at arbitrary positions. The non-singularity of the metric fixes the size of the internal radius $R=8 M G$, and thus the monopole mass is fixed by the geometry of the theory.

\section{Moduli Spaces}

It is clear that any member of the GPS family is given by specifying $N$ points in 3 dimensional Euclidean space and has $3 N$ parameters. But we identify metrics up to a coordinate transformation, and so we subtract 3 parameters for an overall translation and 3 for an overall rotation. In fact the whole situation is more complex.

The configuration space for the dynamics of five dimensional General Relativity [7] is the space of all geometries on a specified four dimensional manifold $M$ which is the orbit space (or superspace)

$$
S(M)=\frac{\operatorname{Riem}(M)}{\operatorname{Diff}(M)}
$$

of the group of diffeomorphisms $\operatorname{Diff}(M)$ acting by coordinate transformation on the space of Riemannian metrics Riem $(M)$. However as some metrics equivalent by diffeomorphism are more equivalent than others $S(M)$ fails to be a manifold. For example a metric exhibiting a continuous or discrete symmetry will be invariant under some subgroup of $\operatorname{Diff}(M)$, i.e. it has non-trivial isotropy group. Such metrics are called symmetric. A metric with no symmetries is called generic. Clearly a neighbourhood of a symmetric point in $S(M)$ cannot be homeomorphic to a neighbourhood of a generic point. Specifically they could even have different dimension (if such existed) and $S(M)$ is a stratified set [7]. Note that this superspace is not the same as Salam and Strathdee's superspace of supersymmetry theory. Now if we wish to make $S(M)$ a manifold then we must factor $\operatorname{Riem}(M)$ by 
$\operatorname{Diff}_{*}(M)$ the subgroup of $\operatorname{Diff}(M)$ which fixes both a point at infinity and its tangent space. Since no isometry has this property, $\operatorname{Diff}_{*}(M)$ acts freely and the resulting quotient space is a manifold. Although $S_{*}(M)$ thus obtained is infinite dimensional, some of these features persist in our finite dimensional case. Interchange of the positions of the monopoles makes no difference, so we factor out by the permutation group on $N$ letters $S_{N}$. By fixing the radius of the internal circle we cannot have one monopole sitting on top of one another and so $S_{N}$ acts freely. Now we wish to factor out by the action on $\frac{\mathbb{R}^{3 N}-\Delta}{S_{N}}$ of $E^{3}$ the Euclidean group in 3 dimensions; $\Delta$ is the diagonal set where two or more of the points coincide. $E^{3}$ does not act freely as some of its elements fix symmetric configurations such as equilateral triangles for $N=3$ or the regular polygons for higher $N$. We can take the union of sets with the same isotropy groups and factor out by $E^{3}$ in each union (or stratum). The strata of dimensions less than $3 N-6$ will then correspond to boundary points in minisuperspace - the set of all geometries corresponding to $N$ monopole metrics. For computations however we will use $\mathbb{R}^{3 N}-\Delta$, which is the analog of $S_{*}(M)$, and call this the moduli space of our solutions.

\section{The Motion of GPS Monopoles}

We now consider the motion of the solitons described above. We use an idea due to Manton [1] namely that the motion is approximated by geodesic motion on the $3 N$ dimensional moduli space equipped with a metric inherited from the kinetic energy functional. This is not a general phenomenon and has to be justified case by case. For the monopoles of SU(2) Yang-Mills-Higgs theory (in the BPS limit), Manton's scheme is justified and this has been implemented recently by Atiyah and Hitchin [1].

Consider the action of simple Kaluza-Klein theory

$$
S=\frac{1}{16 \pi G_{\kappa}} \int d^{5} x{ }^{5} R\left({ }^{5} g\right) \sqrt{{ }^{5} g}+\frac{1}{8 \pi G_{\kappa}} \int d \Sigma \sqrt{{ }^{4} g} K,
$$

$G_{\kappa}=2 \pi R G$ and $K$ is the trace of the second fundamental form of the boundary. We can perform the $4+1$ space-time decomposition of this theory and write the five dimensional line element as

$$
d s^{2}=\left(N^{2}-N_{\mu} N^{\mu}\right) d t^{2}-2 N_{\mu} d x^{\mu} d t-{ }^{4} g_{\mu \nu} d x^{\mu} d x^{\nu} .
$$

The action (4.1) then becomes (after dropping a divergence)

$$
\begin{gathered}
S=\frac{1}{16 \pi G_{\kappa}} \int d^{4} x d t N \sqrt{{ }^{4} g}\left[{ }^{4} R\left({ }^{4} g\right)+K_{\mu \nu} K^{\mu \nu}-K^{2}\right], \\
K_{\mu \nu}=\frac{1}{N}\left(-\frac{1}{2} \partial \frac{g_{\mu \nu}}{\partial t}+N_{(\mu ; v)}\right),
\end{gathered}
$$

where ; denotes the covariant derivative in the 4 metric $g_{\mu \nu}$. So far the lapse function $N$ and the shift vector $N_{\mu}$ are freely specifiable and they play no dynamical role in the theory. This is clearly the case as they may be given any values we choose (at 
least locally) by a coordinate change. $N$ and $N_{\mu}$ are to regarded as Lagrange multipliers and they yield constraints on ${ }^{4} g_{\mu \nu}, K_{\mu \nu}$ :

$$
\begin{gathered}
\sqrt{{ }^{4} g}\left[-{ }^{4} R\left({ }^{4} g\right)+K_{\mu v} K^{\mu v}-K^{2}\right]=0, \\
{\left[K^{\mu v}-K g^{\mu \nu}\right] ; v=0 .}
\end{gathered}
$$

These are the Hamiltonian constraint and momentum constraints which have to be satisfied if the initial data is to be consistent. We can choose $N_{\mu}$ to vanish and $N=1$ - this is called a synchronous reference system, but this will break down at some time in the past or the future [8]. With this choice then,

$$
S=\frac{1}{16 \pi G_{\kappa}} \int d^{4} x d t\left[{ }^{4} R\left({ }^{4} g\right) \sqrt{{ }^{4} g}+\frac{1}{8} G^{\mu \nu \lambda \varrho} \dot{g}_{\mu \nu} \dot{g}_{\lambda \varrho}\right]=\int L d t
$$

where $G^{\mu \nu \lambda \varrho}=\sqrt{g}\left[g^{\mu \lambda} g^{\nu \varrho}+g^{\mu \varrho} g^{\nu \lambda}-2 g^{\mu v} g^{\lambda \varrho}\right]$. So we have a Lagrangian where the scalar curvature term may be regarded as giving minus the potential energy and the term quadratic in $\dot{g}_{\mu \nu}$ as the kinetic energy. This latter term may be regarded as a pseudo-Riemannian metric - the de Witt metric - on superspace [7]. We may view the evolution of our system as a track in superspace. Now suppose that our initial data is given by a four metric corresponding to a GPS multi-monopole solution and that the initial velocity is that induced on superspace by the velocities of the monopoles themselves. We will show that the kinetic energy is positive and that at least locally the potential energy increases in all but a finite number of directions in which it remains constant. The velocity of a point in superspace is an infinitesimal perturbation in ${ }^{4} g_{\mu v}$. However such a perturbation is really a representative of an equivalence class so we must choose a gauge condition. We choose $\delta g_{\mu \nu}$ to be orthogonal to the orbit that $\operatorname{Diff}(M)$ sweeps out through ${ }^{4} g_{\mu \nu}$. By orthogonal we mean with respect to the de Witt metric. This is assured if $\dot{g}_{\mu \nu}$ satisfies the transverse traceless condition. But such tensors are precisely those yielding positive kinetic energy and in addition satisfy the momentum constraints. We will show later that the potential energy is positive semidefinite too and that the finitely many directions in which it is constant correspond to changing the positions of the monopoles. In any case the positive action theorem for ALF spaces ensures that the five dimensional energy is positive and increases about these solutions [9]. So our point in superspace moves in an infinite dimensional well, starting at a point in a finite dimensional local minimum, with velocity tangent to this minimum. But if the velocities are small, then we must stay near this minimum and describe free motion on this finite dimensional space which corresponds to the moduli space. Thus we need consider only finitely many degrees of freedom, and so our effective action is just the kinetic energy term. Hence the equations of motion will show that our point describes geodesic motion on the moduli space with the de Witt metric. However we have so far only checked the momentum constraints. Does our proposed initial data satisfy the Hamiltonian constraint too? Naively it would not appear to do so as the initial data has ${ }^{4} R\left({ }^{4} g\right)=0$, and so we would be forced to take $\dot{g}_{\mu \nu}=0$. However if we regard our perturbation as being the first one in a power series in some small parameter $\lambda$ say, and consider the substitution of this power series

$$
{ }^{4} g_{\mu \nu}=g_{\mu \nu}^{0}+\lambda g_{\mu \nu}^{1}+\lambda^{2} g_{\mu \nu}^{2} \ldots
$$


into both the action and the Hamiltonian constraint, then we find that the lowest order constraint term is

$$
g^{0 \mu \nu} \Delta_{L} g_{\mu \nu}^{2}+G^{\mu \nu \lambda \varrho}\left(g^{0}\right)\left(g_{\mu \nu}^{1} g_{\lambda \varrho}^{1}\right)=0, \quad \Delta_{L}=\square g_{\mu \nu} g_{v \varrho}+2 \operatorname{Riem}_{\mu \lambda \nu \varrho},
$$

which can be satisfied for all $g_{\mu \nu}^{1}$. This is a consequence of $\Delta_{L} g_{\mu \nu}^{1}=0$, which is the condition that the first perturbation is within the moduli space. So to first order the Hamiltonian constraint does vanish and our initial data is consistent.

\section{The Metric on the Moduli Space}

Perturbations within the moduli space satisfy

$$
\Delta_{L} \dot{g}_{\mu \nu}=\dot{g}^{\mu \nu} ;_{\nu}=\dot{g}_{\mu}^{\mu}=0 .
$$

Now recall that ${ }^{4} g_{\mu \nu}$ has self dual Riemann curvature, and so we may use the methods of Hawking and Pope [10] to construct the zero modes of $\Delta_{L}$. For such metrics the anti-self-dual Weyl spinor $\widetilde{\Psi}^{A^{\prime} B^{\prime} C^{\prime} D^{\prime}}$ vanishes, and thus there exists a spin basis comprising two independent covariantly constant primed spinors $\tilde{o}_{A^{\prime}}$, $\tilde{l}_{A^{\prime}}$. Their three linearly independent combinations $\alpha_{A^{\prime} B^{\prime}}^{i}$ are zero modes of the antiself-dual spin 1 operator

$$
\nabla_{A A^{\prime}} \nabla^{A A^{\prime}} \alpha_{B^{\prime} C^{\prime}}^{i}=0, \quad i=1,2,3 .
$$

These three zero modes yield the quaternionic structure of $M$ (that is they are Hyperkahler forms). They correspond to anti-self-dual two forms $J_{\mu \nu}^{i}$ which obey

$$
J^{i} J^{j}=-\delta^{i j} I+-\varepsilon^{i j k} J^{k}
$$

where we raise an index and regard, $J^{i \mu}{ }_{v}$ as a map from $T_{x}(M)$ to $T_{x}(M)$ for each $x \in M$. By the index theorem there are $N$ self dual zero modes of the spin 1 operator that is $N$ self dual Maxwell fields $\Phi_{A B}^{J}$. These are the Maxwell fields associated with each monopole. The products of these fields produce $3 N$ transverse traceless zero modes of the spin 2 operator

$$
h_{A B A^{\prime} B^{\prime}}^{i J}=\Phi_{A B}^{J} \alpha_{A^{\prime} B^{\prime}}^{i},
$$

which satisfy $\Delta_{L} h_{\mu \nu}^{i J}=0$. By similar means one can show that the spectra of the Lichnerowicz operator and the scalar wave operator coincide. On a Euclidean manifold the latter has negative definite spectrum when acting on square integrable functions, and since $R_{\mu v}(g+\delta g)=R_{\mu v}(g)+\Delta_{L} \delta g_{\mu v}$, we see that the potential for our problem is positive semi-definite as stated above. An orthonormal basis for $M$ is given locally by

$$
e^{5}=U^{-1 / 2}\left(d x^{5}+2 \kappa \mathbf{A} \cdot \mathbf{d x}\right), \quad e^{i}=U^{1 / 2}\left(d x^{i}\right),
$$

where $\mathbf{A}$ is given by the non-singular form it takes in each local coordinate patch. If we set [11]

$$
J^{i}=e^{5} \wedge e^{i}-1 / 2 \varepsilon^{i j k} e^{j} \wedge e^{k},
$$

then $d J^{i}=0$ and $* J^{i}=-J^{i}$. Thus we have $[d \delta+\delta d] J^{i}=0$, and the $J^{i}$ are harmonic two forms which are linear combinations of the $\alpha_{A^{\prime} B^{\prime}}^{i}$ above. Similarly it can be 
shown [11] that

$$
F^{J}=\left[U^{-1} U^{J}\right],_{i}\left[e^{5} \wedge e^{i}+1 / 2 \varepsilon^{i j k} e^{j} \wedge e^{k}\right]
$$

are equivalent to $\Phi_{A B}^{J}$. So $h_{m n}^{i J}=J_{m p}^{i} F^{J p}{ }_{n} m, n, p=1,2,3,5$ and we have an explicit construction of all spin two zero modes on $M$. We need these transverse traceless zero modes in order to find the projection of the initial velocities in

$T_{g} \operatorname{Riem}(M)$ onto the section given by our gauge condition.
The velocities are those induced by giving the monopole velocities $\frac{d}{d t}\left(X^{J i}\right)$, so we have

$$
{ }^{4} \dot{g}_{\mu \nu}=\frac{\partial g_{\mu v}}{\partial X^{J i}} \frac{d}{d t}\left(X^{J i}\right)+\text { gauge transformation } .
$$

Now let $\gamma_{\mu \nu}^{i J}=-\frac{\partial g_{\mu \nu}}{\partial X^{J i}}$. Then

$$
\gamma_{55}^{i J}=-U^{-1} U_{, i}^{J}, \quad \gamma_{5 k}^{i J}=2 \kappa U^{-1} A_{k, i}^{J}, \quad \gamma_{k i}^{i J}=U^{-1} \delta_{k i} U_{, i}^{J},
$$

with $2 \kappa \boldsymbol{\nabla} \times \mathbf{A}^{I}=\nabla U^{I} \cdot \gamma^{i J}$ can be shown to be gauge equivalent to $h^{i J}$, and so the projection of ${ }^{4} \dot{g}_{\mu \nu}$ is $-h_{\mu \nu}^{i J} \frac{d}{d t}\left(X^{J i}\right)$. Hence the de Witt metric is given by

$$
\begin{aligned}
d s_{\mathrm{de} \mathrm{Witt}}^{2} & =\frac{1}{64 \pi G_{\kappa}} \int_{M} d^{4} x \sqrt{g}\left(h_{\mu \nu}^{i J} h^{i J \mu \nu}\right) d X^{i I} d X^{i J} \\
& =\frac{1}{128 \pi G_{\kappa}} \int_{M} F^{I} \wedge F^{J} \mathbf{d} \mathbf{X}^{I} \cdot \mathbf{d} \mathbf{X}^{J}=G^{I J} \mathbf{d} \mathbf{X}^{I} \cdot \mathbf{d} \mathbf{X}^{J} .
\end{aligned}
$$

So the moduli space metric is determined solely by the self dual harmonic two forms on $M$. For compact $M, G^{I J}$ encodes information about the topology of $M$, it is related to the intersection matrix of $M$. By Poincaré duality 2 cycles not homologous to zero are dual to harmonic two forms and the integral of the wedge product of these forms over $M$ describes how these two cycles intersect. For noncompact manifolds, there are partial results about $L^{2}$ cohomology which is what we are considering here; however the proofs used do not apply to these metrics even though the results appear to. We must therefore compute $G^{I J}$ explicitly. Locally $F^{J}$ is $d\left(\omega^{J}-U^{-1} U^{J}\left(d x^{5}+\omega\right)\right)$, where $\omega^{J}=2 \kappa \mathbf{A}^{J}$ which is well defined within each patch. We can then regard the integral (5.9) as a sum over integrals over patches in which $F^{J}$ is explicitly known. By Stokes' theorem this can be converted into a sum of integrals over boundaries which have opposite orientation where they meet thus yielding cancellations in the sum. After some algebra we obtain

$$
\int F^{I} \wedge F^{J}=256 \pi^{2} M^{2} G^{2} \delta^{I J},
$$

hence $d s_{\mathrm{de} \text { Witt }}^{2}=\frac{1}{8} \mathbf{d} \mathbf{X}^{I} \cdot \mathbf{d} \mathbf{X}^{J} \delta^{I J}$ and the de Witt metric is flat.

\section{Classical and Quantum Scattering of GPS Monopoles}

We have seen that the de Witt metric computed in the previous section is flat. So our monopoles describe straight lines and neither interact nor scatter. Presumably 
this means that the antigravity condition holds even for non-static monopoles, and yet this does not follow directly. The BPS monopoles [1] also antigravitated, that is, scalar and vector forces cancelled and they could thus be arranged in any static configuration. Yet their motion depended critically on a small parameter when the monopoles were well separated. We can ask then, under what conditions our approximation is valid. Clearly we have relied on the monopole velocities being small, and restricted ourselves to exciting only zero modes of the Lichnerowicz operator $\Delta_{L}$. What is the energy radiated to non-zero modes? The energy flux radiated by a system is proportional to the square of the third time derivative of the quadrupole moment of its matter distribution [8], that is to the sixth power of the velocity. Thus we may neglect this correction and consider our result exact to order (velocity) ${ }^{2}$. In fact we have a check on our result. For the BPS monopoles Manton has performed a calculation of the long range forces between the monopoles when regarded as point particles with scalar and magnetic charges and mass, when in slow motion and thus obtains the asymptotic form of the AtiyahHitchin metric on the moduli space. We can do similarly here. If we expand the retarded potentials for a system of massive particles with electric and scalar charges $G \Sigma_{a}, \kappa Q_{a}$ respectively, then we obtain an effective Lagrangian which is (up to terms quadratic in $v / c$ )

$$
\begin{aligned}
\sum_{a} & \frac{1}{2} m_{a} v_{a}^{2}\left(1+\frac{1}{4} \frac{v_{a}^{2}}{c^{2}}+3 \sum_{b \neq a} \frac{m_{b}}{r_{a b}}\right)+\sum_{a>b}\left(\frac{\sum_{a} \Sigma_{b}-Q_{a} Q_{b}+M_{a} M_{b}}{r_{a b}}\right) \\
& -\sum_{b \neq a} \frac{\sum_{a} \Sigma_{b}}{2 c^{2} r_{a b}}\left(v_{a}^{2}+v_{b}^{2}\right)+\sum_{a>b} \frac{1}{2 c^{2} r_{a b}}\left[\left(\mathbf{v}_{a} \cdot \mathbf{v}_{b}\right)\left(Q_{a} Q_{b}-7 M_{a} M_{b}+\Sigma_{a} \Sigma_{b}\right)\right. \\
& \left.+\left(\mathbf{v}_{a} \cdot \hat{\mathbf{r}}_{a b}\right)\left(\mathbf{v}_{b} \cdot \hat{\mathbf{r}}_{a b}\right)\left(Q_{a} Q_{b}-M_{a} M_{b}-\Sigma_{a} \Sigma_{b}\right)\right]+O\left(1 / r^{2}\right) .
\end{aligned}
$$

(For the electric and gravitational fields, see [8].) If we appeal to the electric magnetic duality of Maxwell theory and assume we can replace the electric charges by magnetic moments in our problem, then we have $M_{a}=M, Q_{a}=2 M, \Sigma_{a}=\sqrt{3} M$ for all $a . \mathbf{r}_{a b}$ is the separation of particles $a$ and $b$. Substituting these values in (6.1), we obtain $\sum_{a} \frac{1}{2} m_{a} v_{a}^{2}\left(1+\frac{v_{a}^{2}}{4 c^{2}}\right)$. That is the kinetic energy of a system of free particles in special relativity to this order. So, as conjectured, the antigravity condition does persist at least at large separation in the non-static case. It is amusing to note that if we demand that identical massive objects with both scalar and magnetic charges antigravitate to this order, then they necessarily possess charges and mass proportional to those of the GPS monopoles.

We can also describe the quantum mechanics of these monopoles. In the Feynman path integral we have to consider paths in $\operatorname{Riem}(M)$ joining two multimonopole configurations. The paths lying wholly in the moduli space will give rise to an effective field theory equivalent to the quantum mechanical particle moving in the moduli space. This is described by the Schrödinger equation in the moduli space metric. So this low energy theory would also be trivial. However paths not wholly in the moduli space could in principle also contribute a term to the effective action equivalent to a potential energy for the quantum mechanical particle. It is 
plausible that the methods of Hawking and Pope [10] could show that the potential would vanish in the Gaussian approximation.

It is not clear that our result could be predicted from the asymptotic form of the metric found above. For example the Atiyah-Hitchin metric tended exponentially to the asymptotic metric. This is a feature of the exchange of massive particles which in Kaluza-Klein theory are those with nontrivial $x^{5}$ dependence. Since these have the U(1) charge associated with the compact dimension, these would have appeared as Yukawa type forces: that is the particles would have to be produced in pairs. In addition such arguments are inherently flat space in nature, and there could also have been curved space effects which would have been polynomial in $1 / r$. We have shown to all orders in $1 / r$ at low velocities that such effects do not occur. Thus it seems that at low energies the GPS monopoles are effectively structureless objects.

\section{Conclusions}

We have described the GPS monopole solutions and justified the application of Manton's scheme for soliton motion to the solitons of a gravitational theory. However we find that for arbitrary numbers of gravitational monopoles the motion is trivial and they pass one another without interacting.

One could speculate on the origins of this result. These monopole solutions have half the maximum number of supersymmetries in five dimensions when regarded as solutions of $N=8$ supergravity [12]. One might be able to obtain qualitative information about the moduli spaces of supersymmetric solitons, and this would be useful in general as many purely bosonic states are supersymmetric when regarded as solutions of bosonic theories admitting supersymmetric extensions. One could also consider adding fermionic zero modes to the perturbations considered here to obtain a moduli space with both bosonic and fermionic coordinates.

Acknowledgements. I should like to thank Dr. N. S. Manton for discussions and Dr. G. W. Gibbons for help and encouragement.

\section{References}

1. Manton, N.S.: A remark on the scattering of BPS monopoles. Phys. Lett. 110 B, 54 (1982); Manton, N.S.: Monopole interactions at long range. Phys. Lett. 154 B, 397 (1985);

Atiyah, M.F., Hitchin, N.J.: Low energy scattering of non-abelian monopoles. Phys. Lett. $107 \mathrm{~A}, 21$ (1985)

2. Ward, R.: Slowly-moving lumps in the $\mathrm{CP}^{1}$ model in $(2+1)$ dimensions. Phys. Lett. $158 \mathrm{~B}, 424$ (1985)

3. Kaluza, T.: Sitzungsber. Preuss. Akad. Wiss. Phys. Math. 121, 996 (1921)

Klein, O.: Quantentheorie und fünfdimensionale Relativitätstheorie. Z. Phys. 37, 895 (1926)

4. Gross, D.J., Perry, M.J.: Magnetic monopoles in Kaluza-Klein theories. Nucl. Phys. B 226, 29 (1983)

Sorkin, R.: Kaluza-Klein monopole. Phys. Rev. Lett. 51, 87 (1983)

5. Gibbons, G.W., Hawking, S.W.: Gravitational multi-instantons. Phys. Lett. 78 B, 432 (1978) 
6. Scherk, J.: Antigravity: A crazy idea? Phys. Lett. 88 B, 265 (1979)

7. Fischer, A.E.: The theory of superspace. In: Relativity, Carmeli, M., Fickler, S.I., Witten, L. (eds.). New York: Plenum Press 1970

8. Landau, L.D., Lifschitz, E.M.: The classical theory of fields. Oxford: Pergamon 1975

9. Gibbons, G.W., Pope, C.N.: Positive action theorems for ALF spaces (1981) unpublished

10. Hawking, S.W., Pope, C.N.: Symmetry breaking by instantons in supergravity. Nucl. Phys. B 146, 381 (1978)

11. Page, D.N.: Private communication; Yuille, A.L., PhD. Thesis, University of Combridge (1980) unpublished

12. Gibbons, G.W., Perry, M.J.: Soliton supermultiplets and Kaluza-Klein theory. Nucl. Phys. B 248, 629 (1984)

Communicated by S.-T. Yau

Received December 13, 1985; in revised form May 5, 1986 